\title{
Methodological recommendations for using the method of work in small groups
}

\author{
M.A. Saydaliyeva ${ }^{1}$, E.B. Atamirzayeva ${ }^{2}$, F.X. Dadaboyeva $^{3}$ \\ ${ }^{1,2,3}$ Teachers of Namangan State University, Uzbekistan
}

Email:ergasheva s@umail.uz

\begin{abstract}
In the present article methodical recommendation on using the method of working in small groups have been reflected, as working in small groups enables the student to develop the skills of cooperation and teach them to solve occurring compromises.
\end{abstract}

Keywords: innovational pedagogy, interactive education, personal-oriented approach, compromise.

\section{INTRODUCTION}

Over the past years, new technologies and teaching methods have been widely used in the educational process. Progress in the educational system brings to the arena a new direction - innovative pedagogy and new pedagogical technologies.New pedagogical technologies in the educational process, first of all, are connected with the personality of the teacher, his pedagogical skills, his spiritual and social level. It seems to us that the main thing in the profession of a teacher is the formation of a professional orientation. This statement not only does not diminish the importance of the teaching profession, but, on the contrary, opens up the possibility for anyone who wants to master it. Refusal from stereotypical classes involves a transition to a new level of training, interactive learning, interactive learning.

Interactive learning - learning, built on the interaction of all students, including the teacher. These methods are most consistent with the personality-oriented approach, since they involve co-education (collective, training in collaboration), and both the student and teachers are subjects of the educational process. The teacher often acts only as the organizer of the learning process, the leader of the group, the creator of the conditions for student initiative. Interactive learning is based on the direct interaction of students with their own experiences and those of their friends, since most of the interactive exercises draw on the experience of the student himself, and not just the educational one. New knowledge, skill is formed on the basis of such experience. Active teaching methods are included depending on the content of the lesson, the initiative of the teacher. One of these teaching methods is work in small groups.Small group classes allow students to acquire collaboration and other important interpersonal skills. In addition, these classes help students (learners) learn how to resolve differences between them.As the group grows, the range of opportunities, experience and skills of its members also expands. Increases the likelihood of a participant whose special knowledge will be useful for completing a group task. The larger the group, the more skills students must show in order to give everyone the opportunity to speak. Such skills require careful training and lengthy practice.

\section{MAIN PART}

The less time is allowed to complete the lesson, the smaller should be the size of the group. Small groups are more effective because they respond more quickly to organizations, work faster, and provide each student with more opportunities to contribute.Interactions within a small group have some features:Groups of two people. In such groups there is a high level of information exchange and less disagreement, but the likelihood of more tension, emotionality and, often, a potential deadlock is also higher. In case of disagreement, none of the participants has an ally.Groups of three people. With such an organization, two stronger individuals can overwhelm a weaker group member. However, groups of three students are the most stable group structures with intermittent shifting coalitions. In this case, it is easier to resolve the differences. Groups with an odd and even number of members. In groups with an even 
number of members, differences are more difficult to settle than in groups with an odd number of members. The odd composition can lead the group out of the impasse or succumb to the majority opinion.A group of five people. This group size seems to be the most satisfactory for educational purposes. A 2: 3 distribution of opinions provides support to the minority. Such a group is large enough to model situations and small enough to involve all participants in the work and personal encouragement. We offer some recommendations for the distribution of students in groups:Teachers are encouraged to place excellent, medium, and poorly performing students in the same group. In diverse groups, apparently, there is more active creative thinking, a more frequent exchange of explanations and a more complete assimilation of perspectives as a result of discussions.

To build constructive relationships between students of different sexes, it is necessary that the composition of each group be as diverse as possible. There are many useful ways to assign students to study groups. The easiest way to randomly distribute is to ask students to pay "first-second." Evens fall into one group, odd ones into another.Some teachers do not change the composition of study groups throughout their studies. It is useful to maintain a stable team long enough for the group to succeed. The disbandment of insufficiently functioning groups is often unproductive, because students do not acquire the skills necessary to solve problems together. Consider adding an adult to the group.

Common problems:

- Among the typical problems of group work that teachers and observers should pay attention to are the following:

- Respect for the rights and opinions of others. Is each member of the group given an equal opportunity to express their opinion?

- Willingness to compromise and cooperation.

- Support for other people.

Do members of the group support those whose position coincides with their own?

- Willingness to listen. Perhaps the members of the group prefer to speak for themselves, rather than listen to the words of others?

- The conflict. If one or more group members have different positions and these positions come into conflict, does the group try to avoid talking about this conflict? Do the members of the group behave as if they agreed with the opposite position? Do they bring controversial issues to an open discussion?

\section{CONCLUSION}

Thus, when working with small groups, the following recommendations should be considered:

1. Ensure that students have the knowledge and skills necessary to complete the work. Lack of knowledge will make itself felt very soon - students will not make efforts to complete the task.

2. Try to make your instructions as clear as possible. It is unlikely that a group will be able to perceive more than one or two even very clear instructions in one go.

3. Allow enough time for the small group to complete the assignment. Decide what to do with groups that will do the job before the rest.

4. Work with small groups should become a rule in your audience, and not a radical, individual departure from the practice of "lecture-retelling."

5. Be mindful of intragroup management issues. If one student is required to report to the group, ensure a fair choice for the speaker.

6. Be prepared for the increased noise typical of collaborative learning.

7. In the process of forming groups, beware of "labeling" students. Heterogeneous groups are generally desirable.

8. Make sure students sit in circles - knee to knee, eye to eye. Each member of the group should easily see the rest. 


\section{REFERENCES}

1. A.I. Artyukhina, V.I. Chumakov. Interactive teaching methods at a medical university. Volgograd. 2011

2. NaumovLB Educational games in medicine. M., 2012

3. Stupina SB Technologies of interactive learning in higher education: educational-methodical manual. - Saratov: Publishing Center "Science", 2009. - 52 p.

4. Azizkhodjaev. New pedagogical technologies. Tashkent 2005 